\title{
Two-Dimensional Fluid Foams at Equilibrium
}

\author{
François Graner \\ Spectrométrie Physique, BP 87, F-38402 St Martin d'Hères Cedex, France; \\ CNRS UMR 5588 et Université Grenoble I \\ e-mail: graner@ujf-grenoble.fr.
}

\begin{abstract}
This is an introduction to fluid foams for non-specialists. After presenting some applications and modeling of foams in general, we focus on ideal two-dimensional fluid foams at equilibrium. We discuss : the interplay of topology, geometry and forces; the central role of pressure and energy; an analogy with 2D electrostatics; the role of disorder; the minimal perimeter problem.
\end{abstract}

\section{Introduction}

There is a place where chemists, engineers, mathematicians and physicists routinely meet: congresses about foams [26, 40, 36]. In fact, fluid foams stimulate truly interdisciplinary fundamental research with a wide range of industrial applications. They have even fascinated some artists. We will try to explain here why foams are at the crossroads of different approaches, and why they are so attractive.

Section 2 presents their main properties and the corresponding applications. Section 3 tries to catch the essence of what a foam is, and how far we should simplifiy it to model it. Section 4 then proceeds by presenting the position of the problem for physicists and mathematicians. In the remaining of the paper, we mostly focus on our own research, which concerns the statistical physics of two-dimensional (2D) foams at equilibrium. Section 5 tries to simplify the problem and reduce it to known results of electrostatics; Sects. 6 and7 7apply it to determinations of the foam's energy and pressure field, respectively; Sect. 8 addresses the physical aspects of the mathematical "minimal perimeter problem". For 3D aspects, which we do not treat here, we refer to excellent existing books: for the mathematical approach, see [20]; for the physical and historical point of view see [19, 35].

The branch of mathematics which deals with the minimal perimeter problem in two or more dimensions is achieving remarkable progresses [20, 17, 18]. Readers interested in the mathematical aspects of foams, especially their specific interplay between topology, geometry and pressure, might read Sects. 4, 5, 6, and 8. These readers can skip Sects. 3 and 7 which are more intended for foam physicists.

However, the point of view we want to present here is completely different. It is the approach of statistical physics: to start from exact detailed properties and deduce average global properties. We have tried to mention in the text which formulas are approximate: this is the case for Sects. 5.2, 5.3, 6.1, 6.2, 7.2, 8.2, 8.3.

The morphology of foams, i.e. the shape of the cellular domains, may be quantified by the techniques presented in the contributions to this volume by Arns et al. (1.2) and by 


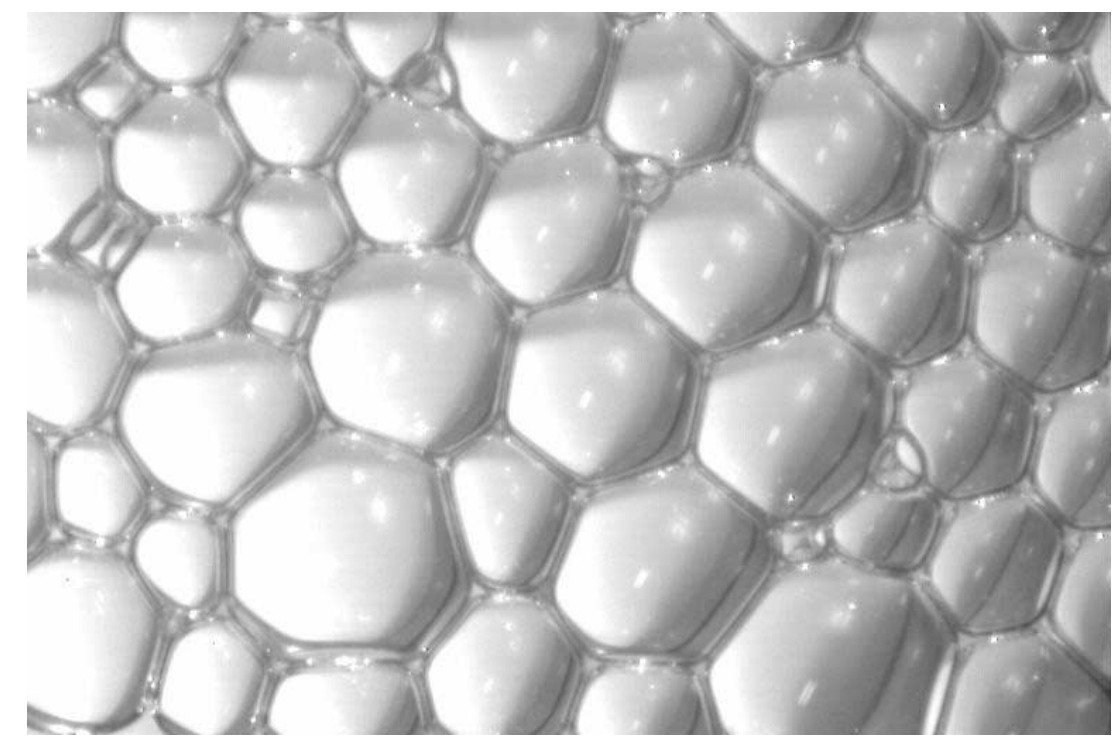

Fig. 1. Example of fluid foam, here the surface of a soap froth. Picture courtesy of S. Courty.

Beisbart et al. (3.2) applying Minkowski functionals on spatially disordered materials. Orientational and tensorial features of patterns are characterised by methods described by P. Soille (3.1) and Beisbart et al. (4.2) in this volume, also useful in the context of foams.

\section{A Wide Range of Properties and Applications}

"Fluid foams" consist of gas bubbles separated by a continuous film of liquid (Fig. 1). Their applications usually combine simultaneously several of their properties, related to their structure, mechanics, chemistry, and surface tension. For reviews, see for instance [19, 39, 3, 12, 13, 25, 34, 11, 14, 33].

\subsection{Structure}

Since the gas occupies most of the volume, foams have a low density, and a high specific surface per unit mass. They can uniformly cover large surfaces. They help in fire-fighting: for oil, which they cover and insulate from air (while water would only fall down, and possibly later boil explosively), and in confined media; foams are much less destructive than water, and the air they contain allows endangered persons to breathe.

Since they can fill volumes with convoluted shapes, they are easy to mould, hence an intermediate step in most processes to prepare solid foams [36]: for cushion stuffing, building materials, bumpers, filters, insulators, catalysts, heat exchangers. Fluid foams fill and can clean pipes or pores, using a small amount of mass; this is essential in nuclear decontamination or chemical pollution where it is necessary to reduce the amounts of 
cleaning residues. They considerably reduce the cost of all fiber treatments: brushing hair, protecting crops; but also waterproofing or dying textiles, being easier and cheaper to remove by drying than bulk water.

\subsection{Mechanics}

Fill a bottle with water, add some dishwashing product, shut it with your thumb, and shake it: the foam damps the shock and your thumb can barely feel it. Such efficient energy dissipation has shock damping applications from demining to gun silencers.

Other police and military applications use foams for confinement: as anti-riots agents, or for preparation and separate storage of $\mathrm{H}$-bombs sub-components. Fluid foams are also useful as temporary insulators: spermicides, thermal insulation of greenhouses during the night, protection of wounds against toxic chemicals, trap for dust in coal mines, prevention of beer oxydation during bottling, prevention of polluant dispersion in soils.

Resistance to pressure is crucial in several applications of foams to oil drilling: as pressurising fluid carrying sand to fracture the rock; to carry away forage residues in under-pressurised regions; to eliminate water; or, by filling empty pores, to prevent vapor, water or gas from leaking through porous rocks.

Note that a foam has a shape, to which it returns after a small mechanical perturbation: it is elastic. Under higher stress, it deforms plastically; under high shear rate, it flows. This triple, elasto-plasto-viscous behaviour, although not specific to foams, is one of their most attractive features. For instance, in shaving or in airplane windshield de-icing, they can be deposed in large amounts on vertical walls thanks to their elasticity, and are later evacuated through the shear produced by the blade or the wind, respectively.

\subsection{Chemistry}

Materials with high affinity for water, especially aqueous solutions, are called "hydrophilic". "Hydrophobic" materials are not wet by water; for instance oil and water do not spontaneously mix. Mixing oil with water, so that rinsing can evacuate the oil, requires cleaning agents, usually "amphiphilic" molecules with a hydrophilic head and a long oily chain made of carbons. Amphiphilic molecules are often also foaming agents, so that foams appear as by-products in cleaning. Neither the presence, nor the stability of the foam seems to improve the quality of cleaning [6]. In that case their presence is a marker of the dose injected, and their destruction marks the presence of impurities.

The amphiphilicity of the foams implies they have an affinity for most materials. Fine tuning their chemical composition leads to selective affinity. This is used in mines to separate ore and useless materials in huge flotation tanks. Foams also can separate colorants by fractional distillation.

\subsection{Model Systems}

In fundamental research, foams are used as model systems. Since they minimise their surface energy by decreasing the area of the bubbles, they are examples of the mathematical "minimum perimeter" problem which we discuss below, and of minimal surfaces 
used in architecture. They are often compared with other cellular patterns : solid foams, biological epithelia or aggregates, ordered pattern like the retina or honeycombs, fracture pattern like in basalts or salt lakes. However, the most useful analogy is with cellular patterns which do have a surface energy and minimise it: grain boundaries in crystals, magnetic domains. Since their characteristics are entirely measurable from images, without any hidden variables, foams are also models for more complex elasto-plasto-viscous materials.

\subsection{Other}

Although the above list of applications is long and could be even longer, foams are often associated with an image of futility. This might originate from their consistence and short life-expectancy. But there might be another reason: foams which are visible in our daily life have less essential applications; or often no applications at all, when they are by-products or polluants.

As light and attractive materials, they are essential to the image of foods and especially drinks like sparkling wines, sodas, or beers, which they protect from oxydation. Without attempting at listing all representation of bubbles and foams in poetry or pictures [19, 9], let us mention "Soap bubbles", by Edouard Manet, or a delicate azulejo in the north-east corner of the garden, in Palacio de la Frontera near Lisbon. They appear in movies, in foam parties, or in both together: "The Party", by Blake Edwards, ends with an unforgettable invasion by a foam provoked by Peter Sellers.

\section{A Generic Model for Fluid Foams}

\subsection{Common Characteristics}

Foams exist if and only if two generic conditions are met:

(i) within each bubble, the matter is conserved; and:

(ii) the energy decreases when the total surface of the walls decreases.

This deserves some discussion and examples.

In a foam the surface energy is an increasing, but not necessarily a linear function of the surface. For instance, the surface tension can be non-uniform due to temperature gradient: Marangoni effect. Or, a crystal usually consists in many small monodomain crystals; the boundaries between these grains actually meet both above conditions: here the surface energy is not uniform nor anisotropic. To the contrary, condition (ii) excludes aggregates of vesicles, although they look like soap bubbles [27]: since their walls do not communicate together, each vesicle has a different tension; the total energy is a function of each vesicle's surface separately, not of their sum.

In addition to this surface energy, the total energy of the foam always contains additional, specific terms which vary from foam to foam. For instance, the repulsion between the electrostatic dipoles of amphiphilic molecules on either side of the film provides a microscopic energy term to stabilise the walls against breakage. 
Minimisation and equilibration of energy requires both phases to be fluid. This includes emulsions, which are fluid-in-fluid foams, like oil in water. This specifically excludes solid foams, granular systems like dry or wet sand.

Due to condition (i), an equation of state relates the pressure and volume of each bubble separately. The surface of the walls decrease and the pressure within each bubble increases until they exactly balance each other. This ensures the mechanical stability of the foam. Two simple cases are often studied: the perfect gas, where the pressure and volume are inversely proportional; and the incompressible emulsion, where the volume is constant and only the pressure varies.

To observe a foam, conditions (i) and (ii) need only be valid during the time required to reach the mechanical equilibrium: typically tens of milliseconds for a soap froth. Almost all real foams violate these conditions at longer time scales. A main cause is wall breakage: foams produced without amphiphilic molecules by violent mixing gas and liquid (froth on sea waves) or chemical reactions (metallic foams) are transient and out-of-equilibrium, and only last a few seconds. A slower phenomenon is drainage of the water under gravity, like in mine flotation tanks; in a beer glass it only takes seconds or minutes. Finally, depending on the solubility of the gas in the liquid, bubbles can lose gas into their neighbours and disappear, resulting in the foam losing its bubbles and coarsening over minutes or hours.

\subsection{The Simplest Foam}

Ideal foam: To catch the essence of the physics common to all foams, we focus on a idealised foam defined as follows.

- Dry foam: the gas occupies most of the foam's volume, while the liquid occupies a very small fraction. In physical experiments this dryness is limited by the stability of the film: typically, in soap froths it can go down to one or two percents. In the ideal foam we take the limit of fluid fraction going to zero.

- Incompressible foam: Although foams are full of gas, we can usually neglect their compressibility: of order $P_{a t m}^{-1}=10^{-5} \mathrm{~m}^{3} . \mathrm{J}^{-1}$, that of a perfect gas. This is much smaller than the effect of the surface tension, characterised by the Laplace overpressure (2) of order of the bubble wall curvature, typically a centimeter, divided by the surface energy, typically $10^{-2} \mathrm{~J} \cdot \mathrm{m}^{-2}$, hence $1 \mathrm{~m}^{3} \cdot \mathrm{J}^{-1}$. Emulsions, which are full of liquid, are of course even less compressible.

- Energy proportional to the surface: this means that surface tension is uniform and anisotropic, and dominates all other contributions to energy. A soap froth with bubbles much larger than molecular sizes often provides a correct example.

- No temperature effect: room temperature does not play any significant role in the structure of a foam, see Sect. 8.1 for order of magnitudes.

Two-dimensional (2D) foams: We chose to focus on two dimensional (2D) foams, although specialists of actual (3D) experiments often object to this choice.

- The first usual objection to 2D foams is their lack of practical applications by themselves. The answer is that they turn particularly useful via their use in fundamental 
research. 2D experiments and simulations are easier to realise and, what is more important, to analyse both qualitatively and quantitatively. Theories are also easier to develop in $2 \mathrm{D}$, before generalisation to $3 \mathrm{D}$, especially since there are more exact results in $2 \mathrm{D}$; this will be addressed in Sect. 4

- The second, more radical objection is that experimental foams are never truly 2D. The answer is that it does not really matter. To understand the physics, we perform truly $2 \mathrm{D}$ simulations and theories, and approximately 2D experiments. An experiment is a correct model of $2 \mathrm{D}$ foam if the energy is proportional to the total perimeter. For instance, in a soap froth between two parallel plates of glass, each line has an energy proportional to its length, 3D effects such as the curvature in the vertical plane appearing only in the value of the proportionality constant. Some 2D foams are stabilised by dipolar interactions without amphiphilic molecules [29]: in Langmuir monolayers [4], magnetic garnets [37], oil/ferrofluid emulsions [7]; they are acceptable model of 2D foams if and only if the dipolar energy is small enough not to affect their behaviour.

\section{Position of the Problem}

From now on, we consider only ideal, dry 2D foams at equilibrium. This section deals with its structure: first the position of the problem, then some directions of research. Most results below come from [15], to which we refer for details.

\subsection{Energy}

An ideal 2D foam consists in $N$ bubbles with fixed areas $\left\{A_{i}, i=1, \ldots, N\right\}$, Fig. 2 . Its energy [41] is simply the sum of wall lengths:

$$
H=\gamma \sum_{0 \leq i<j \leq N} \ell_{i j}=\lambda \sum_{i, j=0}^{N} \ell_{i j}=\lambda \sum_{i=0}^{N} \mathcal{L}_{i}
$$

where $\ell_{i j}$ is the length of the wall between bubbles $i$ and $j, \mathcal{L}_{i}=\sum_{j} \ell_{i j}$ is the perimeter of bubble $i, i=0$ denotes the medium that surrounds the foam, $\ell_{i i}=0$. Here $\lambda$ is the line energy of the interface between water and gas, $\gamma=2 \lambda$ is the line energy of a wall consisting of two interfaces, both in $\mathrm{J}_{\mathrm{m}} \mathrm{m}^{-1}$.

If $\langle A\rangle$ is the average bubble area, note that $H / \lambda\langle A\rangle^{1 / 2}$ is a dimensionless function of the bubbles' shapes only. In this sense, minimising the energy is a purely geometrical problem, universal in the sense that it is the same for any perimeter-minimising system.

\subsection{Local Rules: Plateau}

At equilibrium, the ideal foam obeys the following local rules, stated by Plateau [24]. 




Fig. 2. Example of a 2D foam, here a soap froth between two plates of glass. The line tension $\lambda$ is here twice the usual surface tension between water and gas (here $27.3 \mathrm{mN} / \mathrm{m}$ ) multiplied by the distance between plates $(1 \mathrm{~mm})$. Picture courtesy of O. Lordereau.

Walls: According to Laplace's law, the wall between bubbles $i, j$ has a curvature which balances the 2D pressure difference:

$$
\kappa_{i j}=\frac{P_{i}-P_{j}}{\gamma}
$$

where $\kappa_{i j}=-\kappa_{j i}$ is the algebraic curvature, counted positive when bubble $i$ is convex compared with bubble $j$. As a consequence, along the wall, the curvature is uniform: each wall is an arc of circle.

Vertices: Bubble edges meet in triples at $2 \pi / 3$ angles (Fig. 3). Foams have the topology of three-fold connected networks; four-fold vertices cost more energy and are unstable (Fig.7).

As a consequence of (2), algebraic curvatures of the three edges that meet at the same vertex must add to zero:

$$
\kappa_{i j}+\kappa_{j k}+\kappa_{k i}=\frac{P_{i}-P_{j}}{\gamma}+\frac{P_{j}-P_{k}}{\gamma}+\frac{P_{k}-P_{i}}{\gamma}=0 .
$$

Equation (3) holds for any closed contour crossing more edges. 




Fig. 3. Illustration of Plateau rules, on a $2 \mathrm{D}$ soap froth. Bubble walls are arcs of circles meeting by three at (approximately, since the foam is not ideally dry) $120^{\circ}$ angles. A pentagon is convex and at high pressure, with respect to its neighbours. Picture courtesy of S. Courty [5].

\subsection{What Is the Problem?}

At first sight, the ideal foam appears truly simple. The expression of its energy, (1), is one of the simplest one can dream of. It immediately yields very intuitive consequences, the above Plateau rules, which considerably reduce the number of degrees of freedom necessary to describe the structure: for each vertex, an angle, modulo $2 \pi / 3$; for each side, a length and a curvature.

Now, we give ourselves the area of each of the $N$ bubbles, and the topology of the foam, that is the list of which bubbles share a common edge, and we wonder: what can we say about the structure of the foam, the bubble shape, or the total perimeter? The answer is that there is nothing simple! The disorder inherent to the foam, especially due to the area distribution, but also due to the unavoidable foam boundaries, prevents us from any intuition. There is an intricate interplay between topology, geometry, and pressures, specific to foams, which make them both fascinating and difficult to solve, as the next sections will discuss. 


\section{Electrostatic Analogy}

\subsection{Pressure, Geometry, Topology}

One bubble: Consider a bubble of a foam; let $n$ be its number of sides. It is characterised by a topological quantity which quantifies the deviation from a hexagonal lattice: its topological charge $6-n$. Equivalently, we can say that an $n$-sided bubble is a disinclination bearing a geometrical charge:

$$
q=(6-n) \frac{\pi}{3}=2 \pi-\frac{n \pi}{3} .
$$

Now, circle once counterclockwise around this bubble. What the Gauss theorem states, is here particularly simple: the tangent vector $\hat{t}$ along the bubble edge, which travels around the bubble once counterclockwise, rotates by $2 \pi$. Note $i$ the number of this bubble, $j$ its neighbours. Each side $i j$ is an arc of length $\ell_{i j}$ and of curvature $\kappa_{i j}$, thus contributes $\kappa_{i j} \ell_{i j}$ to this rotation, while each vertex rotates the tangent vector $\hat{t}$ by $\pi / 3$. The Gauss theorem hence links the average curvature of a bubble to its number of sides [23]:

$$
\sum_{j} \frac{1}{\gamma}\left(P_{i}-P_{j}\right) \ell_{i j}=\sum_{j} \kappa_{i j} \ell_{i j}=\left(6-n_{i}\right) \frac{\pi}{3}=q_{i} .
$$

This classical but essential equation is what makes $2 \mathrm{D}$ foams unique. It relates the topology, the geometry (curvatures, lengths), and the pressure (hence forces) in a single bubble. This means for instance that 4-sided or 5-sided bubbles are convex, and their pressure is higher than their neighbours'; see the hatched pentagon in Fig. 4. On the opposite, 7 -sided or 8-sided bubbles are concave and have a low pressure. If this relation was not satisfied, the bubble would not be at equilibrium: the walls would move, and the pressure adapt, until the condition (5) is fulfilled.

Several bubbles: Extension to more than one bubble uses a topological theorem [30, 2]. Consider a contour $C$ which follows only bubble edges and encloses a few bubbles, see

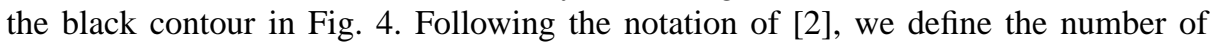
vertices which originate an outward (inward) pointing edge as $v^{+}\left(v^{-}\right)$(Fig. (4); e.g. $v^{+}=0$ if $C$ is the boundary of the total foam, $v^{-}=0$ if $C$ encloses a single bubble. The topological Gauss theorem [30. 2] states that $C$ encloses a total topological charge $\sum_{k \in C}\left(6-n_{k}\right)$ equal to:

$$
\sum_{k \in C}\left(6-n_{k}\right)=\left(6-v^{+}+v^{-}\right),
$$

where $k$ labels the bubbles enclosed by $C$. For instance, if $C$ encloses a single bubble, $v^{-}=0, v^{+}=n$, and one recovers (5).

We can write the Gauss theorem, exactly as we wrote it above for one bubble, by stating that the tangent vector $\hat{t}$ along the contour $C$, which travels around the bubble once counterclockwise, rotates by $2 \pi$. We thus find that: 




Fig. 4. A schematic of a $2 \mathrm{D}$ foam. The black contour $C$ follows bubble edges and encloses a geometrical charge $Q(Q=\pi / 3$ due to the pentagon). Vertices with squares point inwards $\left(v^{-}=5\right)$ and vertices with circular discs point outwards $\left(v^{+}=10\right)$. The dashed contour $C$ crosses bubble edges transversely. Along the contours tangent $\hat{t}$ and normal $\hat{n}$ vectors (arrows) are defined by the right-hand rule. Drawing by Y. Jiang [15].

$$
\sum_{C} \kappa_{i j} \ell_{i j}=\left(6-v^{+}+v^{-}\right) \frac{\pi}{3} .
$$

Combining (6) and (7), we have expressed the geometrical Gauss theorem for a closed contour $C$ which follows the bubble edges $i j$ and encloses a total geometrical charge $Q[15]$ :

$$
\sum_{j} \frac{P_{i}-P_{j}}{\gamma} \ell_{i j}=\sum_{C} \kappa_{i j} \ell_{i j}=Q(C)=\sum_{k \in C} q_{k}
$$


This theorem establishes at a global level the essential relation between topology, geometry and pressure in 2D foams.

Finally, (3) can also be generalised for a contour $C$ crossing the bubble edges perpendicularly, see the dashed contour in Fig (4). The same argument that applied to a contour around a single vertex now yields, for the whole contour, that the curvatures of all edges encountered add to zero:

$$
\sum_{C} \kappa=0 .
$$

\subsection{Continuous Limit}

We now rewrite the above equations as an analogy with 2D electrostatics (Table 1), and discuss its advantages [15].

Table 1. Proposed analogy between foams and electrostatics in two dimensions.

\begin{tabular}{|r||c|c|c|}
\hline & potential & field & charge \\
\hline $\begin{array}{r}2 D \\
\text { electrostatics }\end{array}$ & $\begin{array}{c}\text { potential } \\
V\end{array}$ & $\begin{array}{c}\text { electric field } \\
-\nabla V\end{array}$ & $\begin{array}{c}\text { electric } \\
\text { charge } e\end{array}$ \\
$2 D$ & $\begin{array}{c}\text { pressure } \\
P\end{array}$ & $\begin{array}{c}\text { curvature } \\
\kappa \propto-\nabla P\end{array}$ & $\begin{array}{c}\text { geometrical } \\
\text { charge }(6-n) \frac{\pi}{3}\end{array}$ \\
\hline foams & $P \propto$ & \\
\hline
\end{tabular}

In this analogy, each bubble represents a conducting platelet bearing a charge $q=$ $(6-n) \pi / 3$ distributed along its edges, which (along each link) is uniform at equilibrium because the charge density is proportional to the curvature. The walls are a thin layer of insulating material, which bear a large gradient of pressure (almost a discontinuity). Since we are not interested in the actual pressure within them, we have thus chosen to interpolate the pressure in such a way that $\Delta P=0$ within walls. We introduce the notation $\boldsymbol{E} \equiv-\nabla P$ :

$$
\begin{array}{ll}
\boldsymbol{E} \propto \kappa \hat{n} & \text { (across walls), } \\
\boldsymbol{E}=0 & \text { (within bubbles), }
\end{array}
$$

where $\kappa$ is the curvature of the walls and $\hat{n}$ its outwards normal.

The pressure thus appears as a potential, the curvature as its gradient (Table 1). Let the unit vectors $\hat{n}, \hat{t}$ be the normal and the tangent to $C$, respectively, as drawn in Fig. 4 . In the dry foam limit (see [15] for details), (3]8) can be rewritten under a more familiar continous form: 


$$
\begin{aligned}
& \oint_{C} \boldsymbol{E} \cdot \hat{n} d \ell \propto Q(C), \\
& \oint_{C} \boldsymbol{E} \cdot \hat{t} d \ell=0 .
\end{aligned}
$$

These approximate relations hold for any contour $C$ (not necessarily parallel or perpendicular to edges), although it is easier to visualise (11) on the black contour, $11 \mathrm{~b}$ ) on the dashed one. Although both $[11$, b) look similar, they are physically different, describing respectively an outwards flux through $C$ (which, in $2 \mathrm{D}$, is a line integral instead of a surface integral) and a circulation along $C$. They make the analogy to electrostatics obvious.

\subsection{Examples}

Pressure and curvature fields: A positive $q$ corresponds to a local high pressure. In turn the pressure gradient correlates with the concavity of the bubbles. For illustration, we consider a foam with all bubbles having the same area $A$, that is a honeycomb of side $L$. Take one single "defect" bubble with a geometrical charge $q=(6-n) \pi / 3$ as a germ bubble (disinclination), for instance a regular pentagon, hatched in Fig. 4 The foam consists of concentric shells around this germ. When the foam is sufficiently large, in the $s$ shell, $s \gg 1$, the asymptotic limits of both $\kappa$ and $P$ are approximately :

$$
\begin{aligned}
\kappa(s) & \propto \frac{q}{L s}, \\
P=\gamma \sum_{s} \kappa(s) & \propto P_{0}-\frac{\gamma q}{L} \ln s .
\end{aligned}
$$

Here $P_{0}$ is a constant $e . g$. equal to the pressure $P_{b}$ at the boundary of the foam, and $P$ grows logarithmically with the foam size. Equations (12) are characteristic of $2 \mathrm{D}$ electrostatics.

Dipoles and quadrupoles: Foams thus enter the well-known class of universality of 2D Coulomb interactions, which encompasses e.g. electrostatics, lines of disinclinations, or arrays of vortices. Two opposite charges $-q+q$ (e.g. a pentagon-heptagon pair, Fig. 5a) a distance $d \sim L$ apart constitute a dislocation, that is a dipole of moment $\boldsymbol{p}=q \boldsymbol{d}$, which deforms neighboring bubbles and induces curved edges in the hexagons around it. At a distance $s \gg d$ away from this dipole, the pressure goes approximately as $1 / s$ and the curvature as $1 / s^{2}$ : similar to the dipolar potential in 2D. A dipole can pair with another dipole (Fig.5 5 ) to form a topological quadrupole (Fig.5b), which affects the honeycomb lattice over a much shorter range, the pressure going as $1 / s^{2}$ and the curvature as $1 / s^{3}$.

\section{The Energy of the Foam}

\subsection{Perimeter Increase due to a Defect}

The analogy is deeper and extends to the expression of energy itself. The energy, or sum of perimeters, of a 2D foam is similar to the energy in 2D electrostatics. We can even 


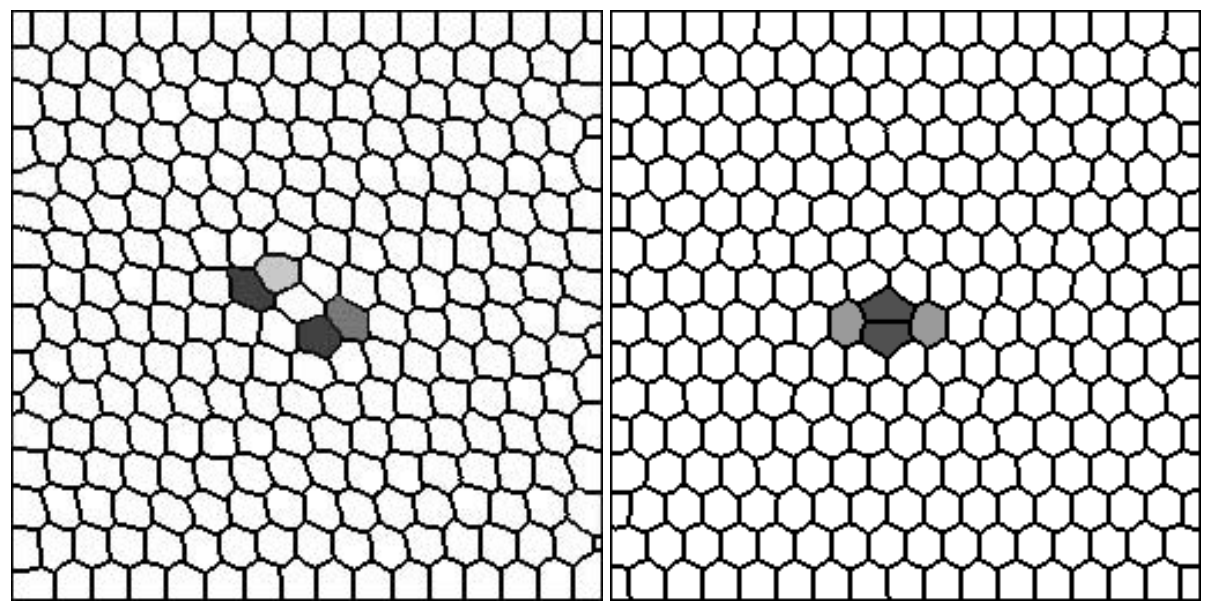

Fig. 5. (a) Simulation of two dipoles in a regular foam with equal areas $(\delta A /\langle A\rangle=0.4 \%)$ and periodic boundaries. Two pentagon-heptagon pairs result in a curvature field in the hexagons around them. (b) An artificially constructed pentagon-heptagon-pentagon-heptagon cluster forms a topological quadrupole, with the rest of the honeycomb lattice undisturbed. Simulation by Y. Jiang [15]

define an energy density for 2D foams. The demonstration applies to honeycomb lattices with only few topological defects, and proceeds as follows.

Energy density: In a honeycomb, when a topological defect induces a curvature in the neighboring edges, the total foam perimeter increases. To estimate this increase, consider two vertices separated by a distance $a$. An arc with curvature $\kappa$ connecting them has a length $\ell=2 \alpha / \kappa$, where $\alpha=\arcsin (a \kappa / 2)$ is half of the subtended angle. The difference between $a$ and $\ell$ is the length increase due to the curvature:

$$
\ell-a \approx \ell\left[\frac{\kappa^{2} \ell^{2}}{24}+O\left(\kappa^{4} \ell^{4}\right)\right] .
$$

As expected, $\ell-a$ is positive and quadratic in $\kappa$. This is the expression of the energy density, proportional to the square of the field.

Energy: By summing over all edges of the foam, and keeping only the leading order when $\kappa \ell \ll 1$, we obtain the increase in energy due to curvature:

$$
H_{t} \approx \gamma \sum_{i<j} \ell_{i j} \times \frac{\kappa_{i j}^{2} \ell_{i j}^{2}}{24}
$$

For instance, around the single topological charge, $\kappa(s)$ decreases approximately as $q / s$ (12). The summation over all curved edges yields the energy cost due to this single topological defect: 


$$
\begin{aligned}
H_{t} & \propto \gamma \sum_{s} s L \kappa^{2}(s) \\
& \propto \gamma L q^{2} \sum_{s} \frac{1}{s}
\end{aligned}
$$

It grows logarithmically with the size of the foam, as expected in analogy with the selfenergy of a 2D electrostatic charge. For a large foam, a single topological charge costs so much energy that it never occurs in real foams.

\subsection{Several Topological Charges}

Consider two charges $q, q^{\prime}$ separated by a (topological) distance $s \gg L$, the interaction energy goes approximately as:

$$
H_{t} \approx-q q^{\prime} \gamma L \ln \left(\frac{s}{L}\right)
$$

It decreases when charges of same sign separate, or charges of opposite sign aggregate. Heptagon-pentagon pairs are more likely than isolated heptagons. This "effective interaction" of topological origin, which previous work has assumed [28] or derived empirically [25], has important consequences. For instance, this interaction could explain the origin of the correlations between bubbles: the side number distribution and the Aboav-Weaire law [28]. As an illustration, in Fig. 6 the heptagon (indicated by a number 7) has two pentagonal neighbors.

This description remains valid even for many charges, as long as the curvature fields they induce are small and can be added by linear superposition. The pressure fields of multiple charges are added linearly too. However, the present calculations assume equal areas; they become more difficult when the area disorder couples with the topological disorder.

\subsection{Relation Between Pressure and Energy}

For a free foam, in any equilibrium state, there exists a relation between the energy and the pressure field. Surprisingly, it is strictly exact:

$$
H=2 \sum_{i=1}^{N}\left(P_{i}-P_{b}\right) A_{i},
$$

where $P_{b}$ is the pressure of the outer fluid at the boundary of the foam. This generalises in $D$ dimensions:

$$
H=\frac{D}{D-1} \sum_{i=1}^{N}\left(P_{i}-P_{b}\right) A_{i} .
$$

There exist different demonstrations [1]; the shortest is probably the following, based on a Legendre transformation, using $P_{b}$ as a Lagrange multiplier [15]. Consider a free foam with fixed pressures, not areas. At equilibrium, the enthalpy, $\mathcal{H} \equiv H-\sum_{i=1}^{N}\left(P_{i}-P_{b}\right) A_{i}$, is extremal. Thus, under dilation, $L \rightarrow \lambda^{D-1} L, A \rightarrow \lambda^{D} A$, the function: 




Fig. 6. Annealed 2D ferro-fluid foam. Foam preparation: An ionic magnetic fluid (aqueous magnetic liquid, black) and oil (white-spirit, grey) do not mix, their surface tension is $15 \mathrm{mN} / \mathrm{m}$. They are trapped together in the $1 \mathrm{~mm}$ space between two parallel Plexiglas plates. A homogeneous magnetic field of $9 \mathrm{kA} / \mathrm{m}$ perpendicular to the plates induces the cellular structure and fixes the bubble areas and wall thickness. The same oil as that fills the bubbles surrounds them. For details see [7]. Annealing procedure: We tilt the Plexiglas plates from the horizontal plane to an angle of $0.1^{\circ}$, inducing a low effective gravity field. Large bubbles drift upwards, small bubbles downwards, resulting in vertical sorting according to size. We then bring the plates back to horizontal, and the bubbles slowly drift and settle. This procedure allows the bubbles to rearrange and explore the energy space to find a lower energy configuration. The picture displays the final stable pattern. Picture size is $10 \mathrm{~cm}$. Picture by E. Janiaud, for details see [15].

$$
\mathcal{H}(\lambda)=\lambda^{D-1} H-\lambda^{D} \sum_{i=1}^{N}\left(P_{i}-P_{b}\right) A_{i},
$$

is extremal at $\lambda=1$; this proves (1718). 


\section{The Pressure Field within the Foam}

The electrostatic analogy emphasises the central role played by the pressure. How can we determine experimentally the pressure field within a 2D foam? We summarise and compare different methods which could be tried.

\subsection{Measurements}

Direct measurements: An advantage of 2D foams is that each bubble is accessible from outside. It is possible to replace one of the glass plates by an array of pressure sensors [F. Elias, private communication]. This method would present no fundamental difficulty, and would yield an unambiguous result, requiring only a compromise between the price of the equipment and the desired precision. The following methods do not use any specific equipment, only image analysis.

Through areas: The measurement of bubble areas yields access to the pressure if stringent conditions are met. The bubble must be compressible and its equation of state known (e.g. a perfect gas). The area variations must result from pressure variations, not from variation of the amount of matter enclosed in the bubble. The pressure of the bubble must be known at least at one time (for instance at equilibrium with atmospheric pressure). Each bubble area must be tracked individually in time. This method has been successfully applied to a 2D soap froth (M. Asipauskas, to be published).

Through coarsening: Conversely, the measurement of bubble area variations applies under a different set of conditions. The area variation is due only to diffusion of matter from one bubble to its neighbour across their wall, resulting in the coarsening of the foam. The pressure difference is related with the flow rate across the wall, itself related with the normal velocity of the foam if each bubble is incompressible.

Through curvatures: By measuring the curvatures from a picture, and using iteratively eqs (2), the pressure $P_{i}$ in each bubble can be determined up to an additive constant $P_{0}$, the pressure in a reference place (a given bubble, or the outer medium). We only need to chose a path which links bubble $i$ with the reference place, and sum the algebraic curvatures of the walls crossed. Two different paths would give the same result: this is readily seen by joining both path to close a circuit, and apply (9) or its continuous equivalent $(10 \mathrm{~b})$. Thus the choice of the path is arbitrary, and the result is unambiguous.

In practice, this method is imprecise and suited only to very dry foams with good image contrast, due to the notorious difficulty of curvature measurements [O. Cardoso, private communication]. In the example of Fig. 6 the error is around $15 \%$ [15]. An improvement could be to measure all curvatures, typically $3 N$ measurements, and try to find the $N$ pressures which best fit the $3 N(2)$. 
Through vertex positions: M.-L. Chabanol obtained a significant progress by noting that vertex positions yield as much information as curvatures. In fact, the vertex positions yield the length of the chord which joins them, which approximates the value of the wall's length. We thus rewrite the essential (5):

$$
\sum_{j} \frac{P_{i}-P_{j}}{\gamma} \ell_{i j}=\left(6-n_{i}\right) \frac{\pi}{3}
$$

It is easy to solve these $N$ linear equations with $N$ unknown $P_{i}, i=1, \ldots, N$. We then obtain approximate values of the $P_{i}$ s, from which we deduce approximate $\kappa_{i j}$ and $\ell_{i j}$. Reinjecting these values of $\ell_{i j}$ in the $N \times N$ equations systems enables an iteration until stable values for pressures, curvatures and lengths are found.

Preliminary tests with C. Monnereau-Pittet and N. Pittet have yielded a quick convergence to surprisingly good results. The method is correct even on images with low contrast, thick walls or bad focus, because vertex positions and topologies are well measured. The method seems operational, and it is easy to include boundaries. It seems to converge to a unique set of solutions. Care is required: since the method does not enforce the rule of $120^{\circ}$ angles, the solution corresponds to a real foam only if the positions fed in the system are the positions of a foam's actual vertices. Applications are in progress: they could concern extremely precise determinations of curvatures, for instance for coarsening studies.

\subsection{Laplacian of the Pressure}

The analogy with electrostatics indicates that an $N \times N$ equations system can be brought back to a single equation. In fact, (10) imply that the Laplacian of the pressure $P$ is proportional to the topological charge. We were thus tempted to conjecture that the topology, along with boundary conditions, uniquely fixes the pressure field [15]. This is not strictly true, since the bubble shapes are not rigidly fixed; in fact, D. Weaire and S. Cox found a counter-example [38]: two patterns, with exactly the same topology and bubble areas, but a different set of pressures, curvatures and lengths.

However, the Laplacian point of view becomes very interesting on larger scales. On a "mesoscopic" scale much larger than one bubble, but smaller than the foam size, the net topological charge of the foam is zero [16]. This implies that:

$$
\text { (mesoscopic) } \Delta P \approx 0 .
$$

The mesoscopic average of $P$ thus has all properties of zero-laplacian fields. For instance, it is a very "smooth" field; minima or maxima of pressure can exist only on the boundary of the foam (like Earnshaw theorem in electrostatics [22]). If the foam is free, i.e. surrounded by an outer medium with uniform pressure (Fig. 6), its pressure is thus roughly uniform. If the foam is in a closed box, its maxima are imposed by the shape of the box; hence, as N. Pittet noticed, the pressure field and hence the coarsening should differ in round, square and hexagonal boxes. If the foam is flowing in a channel, there is a pressure gradient fom the entrance to the exit. This mesoscopic point of view helps considering the foam as a continuous medium [21]. 

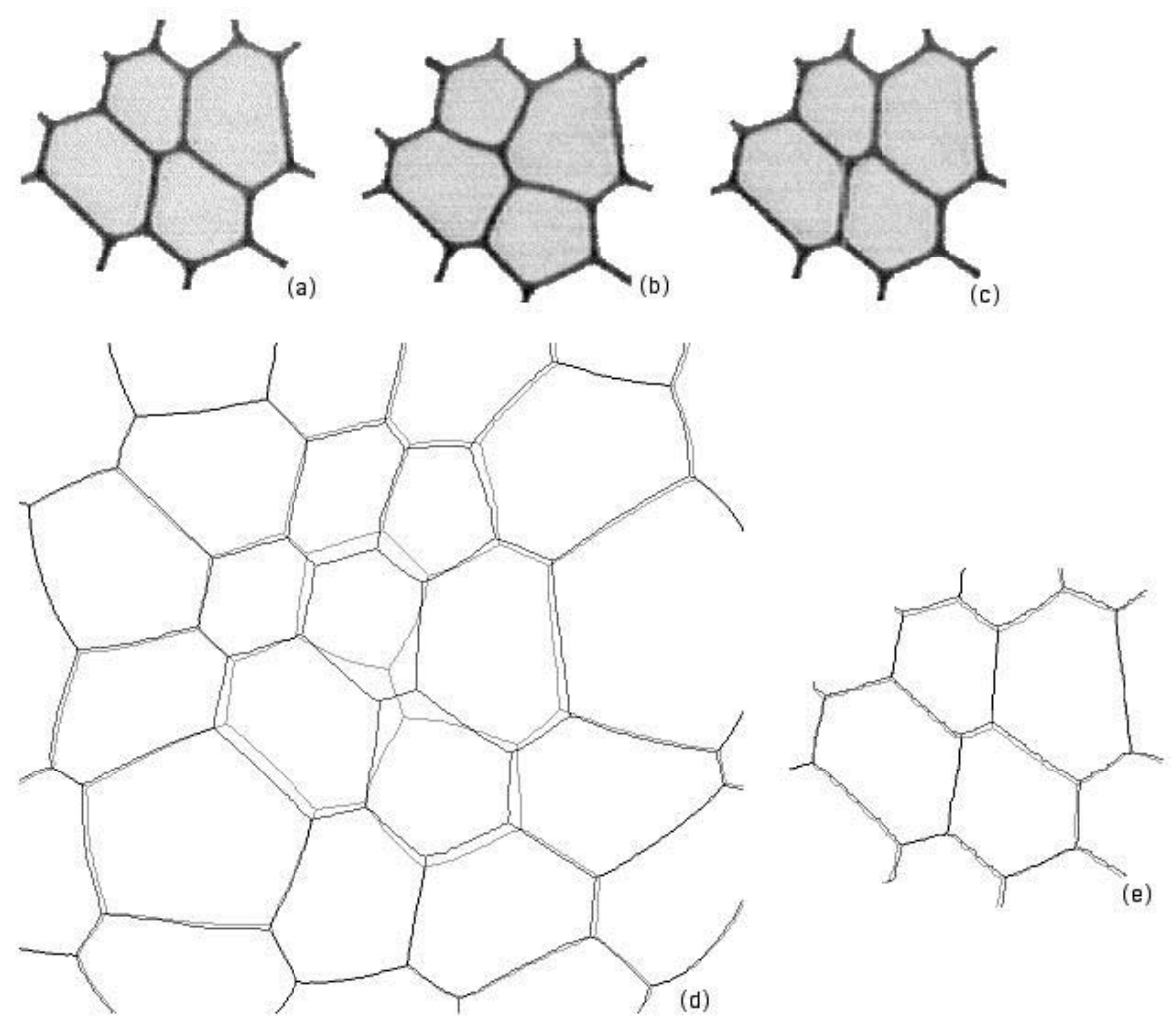

Fig. 7. Artificial side-swapping, or T1 process. We start from an initial equilibrium state (a). A metallic pin placed above the ferro-fluid foam locally channels the magnetic field lines and attracts some ferro-fluid. This enables us to locally move a vertex and bring it closer to the second one, until they merge and form an unstable 4-fold vertex. The foam spontaneously relaxes towards a new equilibrium state with a different topology (b): bubbles have swapped a side and changed their neighbours. Although the energy difference between (a) and (b) was much smaller than our sensitivity, (b) has visibly more regular bubbles, hence likely a lower energy than (a). The skelettised image (d) is the difference between (a) and (b), stressing the range of modifications induced by this T1: typically 3 bubble sizes. Inducing the reverse T1 was possible (c), and we fall back in the same state, see the comparison between (a) and (c) displayed in (e): both (a) and (b) are lasting equilibrium states. However, the reverse T1 was much more difficult: since the 4-fold vertex systematically decayed into configuration (b), we had to nucleate the side we wanted to appear. Hence the energy barrier between (a) and (b) is asymmetric, again suggesting that (b) has a lower energy than (a). Picture by F. Elias. For details see [8]. 


\section{The Minimum Perimeter Problem}

\subsection{Different Equilibrium States}

Topological changes: When the foam is at equilibrium, it remains there. However, the equilibrium can be modified, either by mechanical perturbation such as shear, or by variation of control parameters such as the content of each bubble, through diffusion. If one wall length happens to vanish, four bubbles come in contact. Such four-fold vertex is unstable and decays into one of either possible configuration. The foam spontaneously choses one, likely the one with the lowest energy, and eventually reaches a new equilibrium (Fig. 7). If bubbles have exchanged their neighbours, this elementary topological change is called a side-swapping, or T1 process. A T1 process conserves not only the total charge but also the total dipolar moment, altering only the quadrupolar moment of the foam: it is a current of dipoles.

The energy difference between both equilibrium states is usually much smaller than the barrier which separates them (Fig. 7). In fact, creating a four-fold vertex costs of order the line tension times a wall length, typically $10^{-7} \mathrm{~J}$. The foams with the lowest barrier, namely in Langmuir monolayers, have a cost larger than a $\mathrm{pN} \times \mu \mathrm{m}$, i.e. at least 3 orders of magnitude larger than $k_{B} T$ ( $k_{B} T$ being reached at the scale of the $\mathrm{nm}$, which is the realm of micro-emulsions rather than foams). Thus a T1 never occurs spontaneously under thermal fluctuations, and local energy minima are metastable.

Position of the problem: This raises the question: if we could vary freely the topology, keeping the area distribution fixed, what would be the global energy minimum, i.e. the lowest accessible value of the energy? And what would be the ground state(s), i.e. the corresponding configuration(s)?

This question, called "the minimal perimeter problem", is extremely challenging [20]. Mathematicians are progressing at a quick pace, but exact results still mainly concern small numbers of bubbles, typically $N=2,3$ [18]. However, studying a few bubbles does not help understanding foams with large $N$. Since a topological change can modify the shape of bubbles over a distance of order three bubble sizes (Fig. 7d), the problem is non-local.

In fact, the mathematical result which is the most useful for physicists, is so useful and natural that physicists believed it was obvious: if all bubbles have the same area $A$, the best pattern is a regular hexagonal lattice! The global energy minimum is then $2^{3 / 2} 3^{1 / 4} \lambda \sqrt{A}$. This famous "honeycomb conjecture" is now a theorem, recently proved by Hales [17]. We use this strong result as a starting point for the following approximate, large scale approach [15].

\subsection{Global Energy Minimum (Minimal Perimeter at Free Topology)}

An estimate: If bubbles have different areas, not all bubbles need be hexagons. However, it surprisingly happens that regular bubbles obeying Plateau rules, with $n$ curved edges meeting at $2 \pi / 3$ angles, all have almost the same ratio of perimeter to square root of area: of order $2^{3 / 2} 3^{1 / 4} \approx 3.722 \ldots$, and always larger than $2(\pi)^{3 / 2} / 3 \approx 3.712 \ldots$ [15]. 
We thus suggest that $H$ is close to the energy $H_{h}$ it would have if all bubbles were regular hexagons:

$$
H \approx H_{h}=3.72 \lambda \sum_{i=1}^{N} \sqrt{A_{i}}=3.72 \lambda N\langle\sqrt{A}\rangle,
$$

so that $H_{h}$ is a good approximation of the foam's global energy minimum. This estimate depends only on the area distribution of the bubbles. It depends on $\langle\sqrt{A}\rangle$, while our intuition rather often uses $\langle A\rangle$. If the foam has a boundary, there is an additional term of order $\sqrt{N\langle A\rangle}$.

Tests: We have checked this estimate by examining different examples: simulations and experiments on disordered foams [15], analytical and numerical calculations on periodic foams [10], small clusters with large boundary contributions [32]. The main result which emerges is that the estimate (21) seems consistently correct, its precision being typically the percent. The foam energy is usually slightly higher than $H_{h}$, but surprisingly, we could find ordered foams with a value slightly lower than $H_{h}$, see Sect. 8.3.

In addition, most configurations tend to have an energy close to the global minimum, and it is very difficult to prepare a foam with an energy a few percent higher (Fig. 8). This explains for instance why we could not measure the energy variation after a T1 process (Fig.7). This means that the energy estimate is an acceptable approximation. It is operational, since it depends on the area distribution, which is an intrinsic characteristic of the foam, but not on any particular topology.

\subsection{Ground State Configuration (Perimeter-Minimizing Pattern)}

Difficulty: Since most patterns have almost the same energy, the problem of determining the ground state configuration(s) is subtle. Physical foams come always close to the global energy minimum, not close to a ground state configuration.

Equation (17) indicates that the energy reaches its minimum when the largest bubbles have as low a pressure as possible. It is tempting to use it to determine the ground state configuration. However, this research direction has not yet been successful, to our knowledge [M. Fortes, private communication]. The reason is probably that the pressure field is "smooth", as mentioned above (Sect. (7.2); it might differ from bubble to bubble but these spatial fluctuations remain local and invisible at large scales, hence difficult to analyse in details.

We thus use another, approximate approach, to find the ground state configuration(s), in two opposite extreme cases.

Size-sorting: Consider two bubbles inside a foam, with areas $A_{1}, A_{2}$ respectively. If $A_{1} / A_{2}$ is close to 1 , the total perimeter should be minimal if both bubbles are close to regular hexagons. Both bubbles share an edge, which length is a compromise between $\frac{3.722}{6} \sqrt{A_{1}}$ and $\frac{3.722}{6} \sqrt{A_{2}}$, where $\frac{3.722}{6} \approx \frac{2^{1 / 2}}{3^{3 / 4}} \approx 0.62$ is the side of a regular hexagon of 


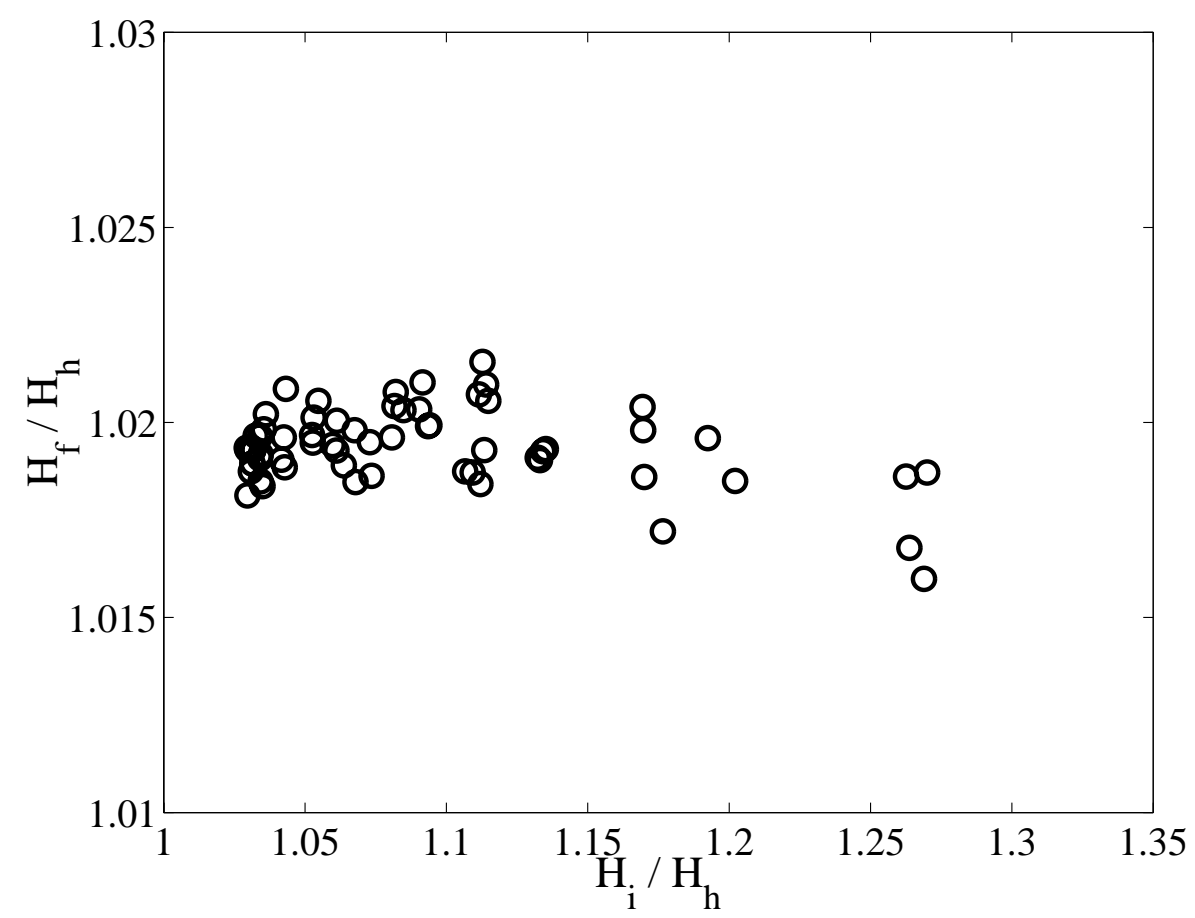

Fig. 8. Test of the estimate for the minimum energy. We simulated out-of-equilibrium, highly deformed foams using the Potts model [15], with a high initial energy $H_{i}$. The foam spontaneously relaxes towards an equilibrium state, with a final energy $H_{f}$. Note the difference between vertical and horizontal scales: although we could reach initial energies $H_{i}$ up to $30 \%$ higher than $H_{h}$, the final energy is systematically the same, barely higher than $H_{h}$. Each symbol corresponds to one foam. Simulations by Y. Jiang [15].

area 1 . We have suggested and checked that, in average, the common edge has a length of order:

$$
L\left(A_{1}, A_{2}\right) \approx \frac{3.722}{6} \sqrt{A_{1}^{1 / 2} A_{2}^{1 / 2}} \approx 0.62\left(A_{1} A_{2}\right)^{1 / 4} .
$$

Thus the cost in total perimeter is lower when bubbles have neighbours of almost the same area. This leads to size-sorting, with bubbles segregating according to their sizes.

We have tried to observe this effect by producing an annealed foam, i.e. a foam as close as possible to the ground state configuration. This is difficult, since the energy barrier between different equilibrium states is larger than the energy differences (Fig.77). We have succeeded by using ferrofluid-foams, easier to manipulate thanks to their high viscosity / low relaxation time. This size-sorting is favoured by a large area distribution, which is here continuous, so that each bubble can find neighbours of almost the same area and be as regular as possible. Such size-sorting is visible on Fig. 6

Size-mixing: On the opposite, when the area distribution is bidisperse, and $A_{1} / A_{2}$ is very different from 1 , bubbles do not remain 6-sided; they rather change their topology. 


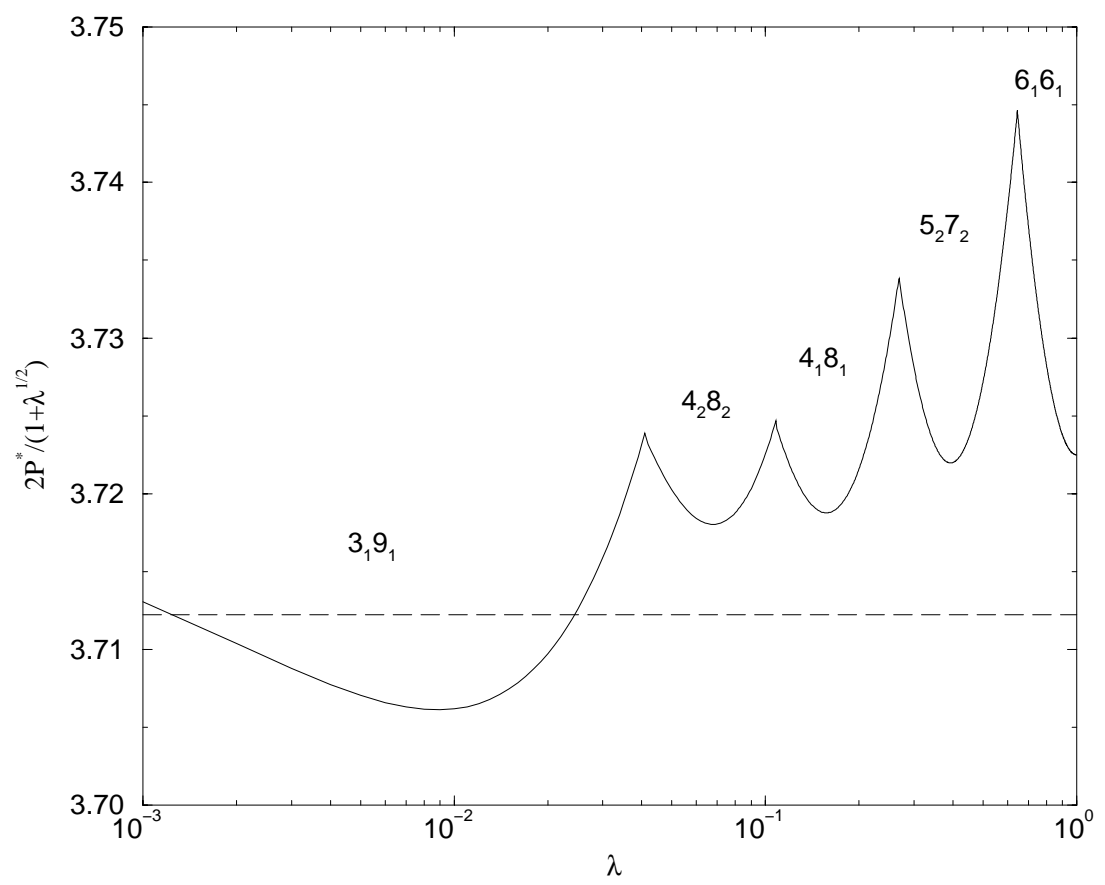

Fig. 9. The best periodic patterns found by Fortes and Teixeira [10]. Two types of cells, of areas $A_{1}=\lambda$ and $A_{2}=1$, are mixed in equal numbers. The vertical axis is twice the perimeter, divided by $\left(1+\lambda^{1 / 2}\right)$ to enable comparison with $H_{h}$ (21). For each value of $\lambda$, only the best pattern found is plotted. The figures indicate the numbers of bubble sides, the indices indicate the number of bubbles per unit cell. For instance $6_{1} 6_{1}$ indicates alternate hexagons; $3_{1} 9_{1}$ indicates an array of large bubbles paving a regular honeycomb, but decorated on each other corner by a small three-sided bubble: surprisingly, this beats the value of regular bubbles, $2 \pi^{3 / 2} / 3$ (dashed line). Graph by P. Teixeira.

Large bubbles ignore small ones, small bubbles decorate the vertices between large ones. Fortes and Teixeira [10] have calculated the energies of different periodic patterns, and looked for the most favorable one, for each given value of $A_{1} / A_{2}$. Their results appear in Fig. 9 Starting from $A_{1} / A_{2}=1$, and decreasing $A_{1} / A_{2}$, they found that the best pattern is successively: alternate hexagons, then mixture of 5- and 7- sided bubbles, then mixtures of 4 and 8 (two types of periodic tilings), and finally of 3 and 9.

Recently, we have found [31] that the periodic tiling of 9-sided and 3-sided bubbles (large bubbles placed in honeycomb lattice, with tiny 3-sided bubbles decorating half of their vertices) beats $H_{h}$, and even slightly beats the value $3.712 \lambda \sum_{i=1}^{N} \sqrt{A_{i}}$ (Fig. 9 ). We are currently investigating this surprising result. 




Fig. 10. A rather exotic $2 \mathrm{D}$ foam! This is a true experiment, actually a ferro-fluid foam. Do you think the 0 -angle (i.e. round) bubble can be at equilibrium? And the 1-angle bubble? Picture courtesy of C. Flament.

\section{Conclusion}

We have tried here to show how foams link mathematical properties, such as topology and curvature, with physical quantities such as energy and pressure (Sect. 5.1). We have adopted the point of view of statistical physics, linking the apparently simple properties of each bubble (Sect.4) with the global behaviour of a disordered foam.

We have considered here an ideal 2D foam (Sect. 3.2) at equilibrium, where each bubble has a given constant area. We have tried to show the central role of the energy and pressure field. We can estimate both on large scales without knowing microscopic details. The energy is approximately a function of the area distribution only, and more precisely on $\langle\sqrt{A}\rangle$ 21); it is almost equal to the perimeter it would have if each bubble had a regular shape, and can even be slightly lower (Sect. 8.3). The pressure resembles a field with zero laplacian (Sect.7.2), as we could understand using an analogy with 2D electrostatics (Sect. 5). Both quantities are also related together (Sect.6.3).

We currently try to open perspectives into three directions: actual 3D foams; mechanical properties of foams out of equilibrium; and more complex fluids such as emulsions and elastic-plastic-viscous materials. We hope to have shown the triple interest of foam research: as a truly fascinating interdisciplinary field, as a carefully-chosen model system, and for their own applications. 


\section{Acknowledgments}

The results presented here have been derived together with M. Aubouy, S. Courty, F. Elias, J.A. Glazier, Y. Jiang. I would also like to acknowledge the collaboration with M. Asipauskas, O. Cardoso, M.-L. Chabanol, S.J. Cox, R. Delannay, B. Dollet, C. Flament, M. Fortes, S. Ifadir, E. Janiaud, O. Lordereau, C. Monnereau-Pittet, N. Pittet, P. Teixeira, M.F. Vaz. Thanks are also due to G. Debregeas, K. Kassner, N. Kern, J. Lajzerowicz, K. Mecke, F. Morgan, D. Weaire for useful discussions. D. Weaire and F. Elias critically read the manuscript. L. Mousson, from the Association des Buveurs d'Orges (www.space.ch/abo), was an endless source of information about beer foams.

\section{References}

1. Aref, H., D. Vainchtein (2000): 'The equation of state of a foam', Phys. Fluids, 12, pp. 23-28 contains two demonstrations. Two simpler demonstrations were then successively proposed by us, in [15], and by Fortes, M. (2001): 'The surface energy of finite clusters of soap bubbles', Phys. Fluids, 13, pp. 3542-3546. Note that it was presented as an already old conjecture in Ross, S. (1969): 'Bubbles and Foam, Ind. Eng. Chem., 61, p. 48 sqq.

2. Aste, T., D. Boosé, N. Rivier (1996): 'From one bubble to the whole froth: A dynamical map', Phys. Rev. E 53, 6181-6191.

3. Aubert, J., A. Kraynik, P. Rand (1986): 'Les mousses aqueuses', Pour la Science Juillet 1986 pp. 62-71

4. Berge, B., A. Simon, A. Libchaber (1990): 'Dynamics of gas bubbles in monolayers', Phys. Rev. A 41 pp. 6893-6900.

5. Courty, S. (2001): Solides bidimensionnels à la surface de l'eau: étude mécanique et optique, $\mathrm{PhD}$ dissertation, University of Grenoble, unpublished.

6. (2001): 'Décapants pour four', Que Choisir mars 2001, 380, pp. 53-57.

7. Elias, F., C. Flament, J.-C. Bacri, O. Cardoso, F. Graner (1997): 'Two-dimensional magnetic froth: Coarsening and topological correlations', Phys. Rev. E, 56, pp. 3310-3318.

8. Elias, F., C. Flament, J. A. Glazier, F. Graner, Y. Jiang (1999): 'Foams out of stable equilibrium: cell elongation and side-swapping', Phil. Mag. B 79, pp. 729-751.

9. Emmer, M. (1991): Bolle di saponi: un viaggio tra arte, scienzia a fantasia (La Nuova Italia Editrice, Scandicci, Firenze).

10. Fortes, M., P. Teixeira (2001): 'Minimum perimeter partitions of the plane into equal numbers of regions of two different areas', Eur. Phys. J. E 6, 131-138.

11. de Gennes, P.-G. (2000): Les mousses, Cours au Collège de France, Paris, unpublished.

12. Glazier, J.A (1989): Dynamics of Cellular Patterns, Ph.D. dissertation, University of Chicago, unpublished.

13. Glazier, J.A., D. Weaire (1992): 'The kinetics of cellular patterns', J. Phys.: Cond. Matt. 4, pp. 1867-1894.

14. Graner, F. (2001): 'La mousse', La Recherche, 345, pp. 46-49.

15. Graner F, Y. Jiang, E. Janiaud, C. Flament (2001): 'Equilibrium states and ground state of two-dimensional fluid foams'. Phys. Rev. E, 63 pp. 011402/1-13

16. Graustein, W.C. (1931): 'On the average number of sizes of polygons in a net', Annals of Math. 32, pp. 149-153. The demonstration is based on Euler theorem, applied here to a non simply- convex foam (e.g. on a torus) [12, 25]. 
17. Hales T. C. (2001): 'The honeycomb conjecture' Discrete Comput. Geom. 25, pp. 1-25; see also Science News, July 24, 1999; Klarreich E., American Scientist 88, March-April 2000, 152.

18. Hutchings, M., F. Morgan, M. Ritoré, A. Ros (2000): 'Proof of the doublebubble conjecture', Electron. Res. Announc. Amer. Math. Soc., 6 pp. 45-49, www.ugr.es/ ritore/bubble/bubble.htm. See also Morgan, F. (2001): 'Proof of the double bubble conjecture' American Mathematical Monthly, 108 (March 2001), pp. 193-205.

19. Hutzler, S., D. Weaire (1999): Physics of Foams (Oxford University Press, Oxford).

20. Morgan F. (2000): Geometric Measure Theory: a Beginner's Guide, revised 3rd ed. (Academic Press, Boston).

21. Jiang, Y., M. Asipauskas, J.A. Glazier, M. Aubouy, F. Graner (2000): ‘Ab initio derivation of stress and strain in fluid foams', in [40], pp. 297-304.

22. Landau, L.D., E.M. Lifschitz (1973): Teoriya Polia (Nauka, Moscow); english translation: The classical theory of fields, fourth revised edition, 2000 (Reed Elsevier, Oxford), chapter 5, p. 95.

23. von Neumann J. (1952): 'Discussion', in: Metal Interfaces, ed. C. Herring (American society for metals, Cleveland Ohio) pp. 108-110, quoted e.g. in [12, 13].

24. Plateau, J (1873): Statique Expérimentale et Théorique des Liquides Soumis aux Seules Forces Moléculaires (Gauthier-Villars, Paris).

25. Rivier, N. (1993): 'Order and disorder in packings and froths'. In: Disorder and granular media, eds. D. Bideau, A. Hansen, (Elsevier Science Publ., Amsterdam), pp. 55-102.

26. Sadoc J.-F., N. Rivier eds. (1999): Foams and Emulsions, Proceedings of 1997 summer school, Cargèse, France (Nato ASI series E, Kluwer, Dordrecht).

27. Sandre, O. (1999): unpublished pictures.

28. Schliecker G., S. Klapp (1999): Europhys. Lett., 48, p. 122.

29. Seul, M., D. Andelman (1995): 'Domain Shapes and Patterns: The Phenomenology of Modulated Phases', Science 267 pp. 476- 483.

30. Smith, C.S (1952): 'Grain shapes and other metallurgical applications of topology', in: Metal Interfaces, ed. C. Herring (American society for metals, Cleveland Ohio) pp. 65-108. Smith's notations $E_{0}, E_{b}$ correspond respectively to $v^{+}$and $v^{+}+v^{-}$, respectively.

31. Teixeira P., F. Graner, M. Fortes (2002): 'Mixing and sorting of bidisperse two-dimensional bubbles', preprint.

32. Vaz M.F., F. Graner, M. Fortes (2002): 'Energy of free clusters of bubbles”, preprint.

33. Vignes-Adler, M., F. Graner (2002): 'La vie éphémère des mousses', Pour la Science, 293, pp. 48-55.

34. Weaire, D. (1995): De la bulle à la mousse', La Recherche 273 (mars 1995) pp. 246-252.

35. Weaire, D. ed. (1997): The Kelvin problem (Taylor and Francis, London).

36. Weaire, D., J. Banhart (1999): Foams and Films, Proceedings of 1999 international workshop on foams and films, Leuven, Belgium (MIT Verlag, Bremen).

37. Weaire, D., F. Bolton, P. Molho, J.A. Glazier (1991): 'Investigation of an elementary model for magnetic froth', J. Phys.: Cond. Matt. 3, pp. 2101-2114.

38. Weaire, D., S.J. Cox, F. Graner (2002): 'On the non-uniqueness of foam equilibrium geometry', Eur. Phys. J. E, to appear.

39. Weaire, D., N. Rivier (1984): 'Soap, Cells and Statistics - Random Patterns in Two Dimensions'. Contemp. Phys. 25, pp. 59 -99.

40. Zitha, P., J. Banhart, G. Verbist (2000): Foams, Emulsions and their Applications, Proceeding of the Eurofoam 2000 conference, Delft, Netherlands (MIT Verlag, Bremen).

41. We use the word "energy" throughout the paper. We do not distinguish here between equations such as (1), where $H$ is a functional of the coordinates, hence strictly speaking a hamiltonian; and such as [17), where it is a value, hence an energy. Moreover, since the 